\title{
Estimating the Vaccine Effectiveness Against Serotype 3 for the 13-Valent Pneumococcal Conjugate Vaccine: A Dynamic Modeling Approach
}

\author{
Aaron Lucas ${ }^{1}$, Michele Wilson ${ }^{1}$, Heather L. Sings ${ }^{2}$, Sarah Pugh ${ }^{2}$, Dylan Jones ${ }^{3}$, Raymond Farkouh ${ }^{2}$, \\ Bradford Gessner', Matthew Wasserman ${ }^{4}$, * \\ ${ }^{1}$ RTI Health Solutions, Research Triangle Park, United States \\ ${ }^{2}$ Pfizer Inc., Collegeville, United States \\ ${ }^{3}$ Pfizer Ltd. Walton Oaks, United Kingdom \\ ${ }^{4}$ Pfizer Inc., New York, United States
}

Email address:

matt.wasserman@pfizer.com (M. Wasserman)

${ }^{*}$ Corresponding author

\section{To cite this article:}

Aaron Lucas, Michele Wilson, Heather L. Sings, Sarah Pugh, Dylan Jones, Raymond Farkouh, Bradford Gessner, Matthew Wasserman. Estimating the Vaccine Effectiveness Against Serotype 3 for the 13-Valent Pneumococcal Conjugate Vaccine: A Dynamic Modeling Approach. International Journal of Infectious Diseases and Therapy. Vol. 4, No. 4, 2019, pp. 56-66. doi: 10.11648/j.ijidt.20190404.12

Received: October 17, 2019; Accepted: November 14, 2019; Published: December 6, 2019

\begin{abstract}
Background: The 13-valent pneumococcal conjugate vaccine (PCV13) is the only PCV licensed to protect against serotype 3 in children. However, conflicting estimates exist of PCV13's direct and indirect protection vaccine effectiveness (VE) for serotype 3. Objective: Our study examined the of PCV13 for serotype 3 using different assumptions for PCV13 direct and indirect $\mathrm{VE}$ to model trends in serotype 3 invasive pneumococcal disease (IPD) and comparing these to observed data from the United Kingdom (UK). Methods: A dynamic transmission model of the spread of pneumococcal carriage and development of IPD was used to fit pre-PCV13-modeled IPD incidence with observed data. To allow for comparison across scenarios, postPCV13-modeled IPD incidence was fit to observed data using assumptions for three different scenarios: (scenario 1) serotype 3 as a nonvaccine serotype, (scenario 2) VE against serotype 3 IPD of 63.5\% based on a recent meta-analysis, and (scenario 3 ) a model-estimated VE against serotype 3. Results: Post-PCV13 introduction, modeled 2017 and average annual serotype 3 IPD incidence were within $20 \%$ and $59 \%$ of observed values for scenarios 2 and 3, respectively, but deviated by $>100 \%$ for scenario 1 . For adults aged $\geq 65$ years, modeled 2017 IPD incidence in scenario 1 differed from observed data by $16 \%$ versus roughly $8 \%$ in scenarios 2 and 3. Conclusions: Observed data do not support a scenario of no serotype 3 VE, but rather a combination of direct protection among vaccinated children and a lower level of indirect protection among older adults. Policymakers should consider transmission dynamics when examining VE against covered serotypes.
\end{abstract}

Keywords: Pneumococcal Pneumonia, Vaccine, Dynamic Transmission Model, Invasive Pneumococcal Disease

\section{Introduction}

In 2000, a 7-valent pneumococcal conjugate vaccine (PCV7) was licensed to target pneumococcal disease due to the most common circulating serotypes at the time $(4,6 \mathrm{~B}$, 9V, 14, 18C, 19F, and 23F). Subsequently, 10-valent PCV (PCV10) and 13-valent PCV (PCV13) vaccines were licensed. PCV10 and PCV13 targeted the same serotypes as PCV7 in addition to three $(1,5$, and $7 F)$ or six $(1,3,5,6 \mathrm{~A}$,
7F, and 19A) additional serotypes, respectively. Both were licensed based on World Health Organization (WHO) recommendations whereby approval of new PCVs was based on the demonstration of immunologic noninferiority to PCV7. These PCVs have been highly effective in reducing incidence of diseases such as invasive pneumococcal disease (IPD), pneumococcal pneumonia, and acute otitis media due to the vaccine serotypes [1].

PCVs reduce the burden of pneumococcal disease through both direct and indirect protection $[2,3]$. The latter occurs by 
reducing nasopharyngeal carriage acquisition or density among vaccinated persons (primarily children) and thus reducing transmission of vaccine serotypes to unvaccinated persons.

Through these mechanisms, PCV13 has led to substantial reductions in IPD globally since its introduction [4]. However, although PCV13 is the only licensed PCV that contains serotype 3 in its formulation, authors have debated the presence and degree of vaccine effectiveness (VE) against this serotype [4, 5]. Given that there was no prelicensure efficacy study for PCV13 in infants, all estimates of PCV13 VE against IPD have been based on realworld observational studies, which have often found different estimates for direct or indirect PCV13 protection against serotype 3 by geography and time [3, 6-8]. For example, after the United Kingdom (UK) introduced PCV13 into the routine pediatric immunization schedule in 2010, vaccine-targeted serotype IPD incidence decreased for PCV13 serotypes other than 19A or plateaued for serotype 19A over the first 4 years following PCV13 introduction in both children and unvaccinated adults [9]. However, beginning in 2014, serotype 3 IPD incidence began to increase in both age groups, while other vaccine-type IPD incidence kept decreasing [5].

There is a large body of evidence from both randomized controlled trials and observational studies that PCV13 provides direct protection against serotype 3 in vaccinated children and adults. A recent meta-analysis of observational studies in infants [10] estimated VE against serotype 3 IPD of $63.5 \%$ (95\% confidence interval, $37.3 \%-89.7 \%)$. In a study funded by the European Centre for Disease Prevention and Control (ECDC), PCV13 VE for serotype 3 IPD was $70 \%$ (95\% confidence interval [CI], 44\%-83\%) for $\geq 1$ dose and $57 \%(95 \% \mathrm{CI}, 5 \%-81 \%)$ for children who were fully vaccinated [11]. In post-hoc analyses from a randomized controlled trial in The Netherlands [12], VE against serotype 3 was $60.0 \%$ (95\% CI, 5\%-85\%) for chest X-ray-confirmed community acquired pneumonia (CAP) and 61.5\% (95\% CI, $18 \%-83 \%$ ) for clinical CAP in the modified intention-to-treat population [13]. Finally, a recent meta-analysis of three studies in adults also found that PCV13 had a VE against serotype 3 for hospitalized CAP of $52.5 \%$ (95\% confidence interval, $62 \%-76 \%$ ) [14]. However, there have also been published case control studies in children that have shown limited effectiveness against serotype 3 IPD, with some suggesting that VE against serotype 3 IPD wanes over time [1]. In addition, and as noted in a previous review of PCV13 impact on serotype 3 IPD in children [10], some prelicensure clinical trials showed that the immune response for serotype 3 following the booster dose was not increased above the levels seen after the infant vaccination series, suggesting potential hyporesponsiveness [16].

More problematic is the debate over the existence of indirect protection from PCV13 against serotype 3. As evidence of indirect protection among older unvaccinated persons, a study published by the ECDC [17, 18] found a statistically nonsignificant reduction in serotype 3 IPD incidence in older unvaccinated adults across six PCV13 settings through 2014, followed by an increase through 2017 , resulting in an overall $12 \%$ increase comparing 2017 to 2009 . Conversely, in PCV10 countries, serotype 3 IPD incidence steadily increased from the time of PCV10 introduction, with a 56\% increase in serotype 3 IPD comparing 2017 to 2009 . In contrast, a randomized controlled trial of PCV7 versus PCV13 in children found no efficacy against serotype 3 carriage, although confidence limits were wide enough to allow for a potential effect [19].

In sum, some investigators $[20,21]$ have concluded that PCV13 has no direct or indirect protection against serotype 3, classifying the serotype as a nonvaccine serotype (NVT). For example, a recent modeling exercise by the Joint Committee on Vaccination and Immunization categorized serotype 3 as an NVT in estimating the impact on removing a priming dose in infant vaccination [22]. Furthermore, a number of costeffectiveness studies also assume PCV13 provides no protection against serotype 3 [23-25]. If such an assumption is incorrect, it will underestimate the benefit of PCV13 vaccination. In this context, it is important to separate direct from indirect effects since a vaccine may provide the former without the latter. In this circumstance, effectiveness or efficacy studies may demonstrate direct protection while population-based surveillance shows no impact among unvaccinated age cohorts, or even target vaccine groups, if coverage remains sufficiently low.

The goal of the current study was to evaluate the validity of assumptions surrounding the VE of PCV13 against serotype 3 . To do this, we compared the prospective trend in serotype 3 IPD incidence post-PCV13 introduction under different VE assumptions, using the observed UK IPD surveillance data as the backbone of the calculations. Many researchers have used mathematical models to carry out experiments, such as this one, that are impractical to conduct in the real world [26-28]. In carrying out this study, we used a previously published and validated model [29] of pneumococcal carriage and IPD that was calibrated to the UK.

\section{Methods}

\subsection{Model Overview}

To assess PCV13 VE against serotype 3 in the UK, we adapted a previously published dynamic transmission model that simulates the spread of pneumococcal carriage and development of IPD in a population over time [29]. The model stratifies individuals by the presence or absence of pneumococcal carriage, vaccine status, and age group. PCV13 vaccination in the UK, based on a $2+1$ schedule (at 2 , 4 , and 12 months of age), is captured by transitioning eligible age groups through vaccine dose compartments based on dosing schedule and adherence.

In the model, individuals who acquire carriage may subsequently develop IPD. Entering a vaccine dose compartment protects against developing IPD by reducing 
the probability of developing IPD $\left(V E_{I P D}\right)$ or reducing the probability of acquiring carriage $\left(V E_{C}\right)$ when exposed to a particular serotype for the duration of protection within the model. The reduction in circulating carriage afforded by the vaccine's $V E_{C}$ lessens the probability of acquiring carriage for unvaccinated adults as well. The model tracks carriage acquisition, carriage duration, and development of IPD over time based on the individual's vaccination status and the population-level carriage prevalence for each serotype (force of infection). On the basis of Wasserman et al. [29], individuals who acquire serotype 3 carry for an average duration of 6.2 weeks and have a probability of IPD given carriage of nine cases per 100,000 acquisitions.

The model includes a fixed set of inputs derived from the published literature [29]. Given these inputs, the model estimates a set of unknown (calibrated) inputs by matching modeled IPD incidence as closely as possible to observed IPD incidence in the UK across five serotype groups (grouped PCV7 serotypes; serotype 3; serotype 19A; grouped serotypes 1, 5, 7F, and 6A; and grouped non-PCV13 serotypes) and seven age groups $(0-<2,2-4,5-17,18-34,35-$ 49, 50-64, and 65 years) [[5]]. Although some authors consider 6A a PCV7 serotype due to cross-reactivity with 6B, for the purposes of this analysis, 6A was considered a PCV13 serotype.

The calibration procedure used to estimate the unknown parameters has been described previously [29]. Briefly, the model estimates a prevaccine-era "steady state" by solving a set of linear equations to calculate the force of infection parameters for each serotype and age group to initialize the model. The model then uses a simulated annealing approach to randomly draw values for the unknown (calibrated) parameters within certain bounds. The model is then run forward over all years of available IPD surveillance (in this case, 2001 to 2017) The calibration procedure is then repeated for a given number of iterations with the goal of minimizing the sum of squared deviations of the resulting yearly IPD incidence values produced from the model as compared with the actual IPD surveillance values by age and serotype group.

Table 1 shows the fixed and calibrated inputs for the $V E_{C}$ and $V E_{I P D}$ for each modeled serotype group excluding serotype 3. Details on other input parameter estimates can be seen in Supplementary Table 4.

Table 1. PCV13 Vaccine Effectiveness Against Serotype Groups.

\begin{tabular}{|c|c|c|c|c|c|c|}
\hline \multirow{2}{*}{ Parameter } & \multicolumn{3}{|c|}{ Direct Protection $\left(V E_{I P D}\right)$} & \multicolumn{3}{|c|}{ Indirect Protection $\left(V E_{c}\right)$} \\
\hline & First Priming Dose & Second Priming Dose & Booster Dose & First Priming Dose & Second Priming Dose & Booster Dose \\
\hline Serotype 19A & $53 \%$ & $75 \%$ & $74 \%$ & Calibrated & Calibrated & Calibrated \\
\hline Serotypes $1,5,7 F, 6 A$ & $85 \%$ & $94 \%$ & $93 \%$ & Calibrated & Calibrated & Calibrated \\
\hline PCV7-covered serotypes & $56 \%$ & $79 \%$ & $93 \%$ & Calibrated & Calibrated & Calibrated \\
\hline NVTs & $0 \%$ & $0 \%$ & $0 \%$ & $0 \%$ & $0 \%$ & $0 \%$ \\
\hline
\end{tabular}

IPD = invasive pneumococcal disease; NVT = nonvaccine serotype; PCV = pneumococcal conjugate vaccine; PCV13 = 13-valent PCV; VE = vaccine effectiveness; VEC = vaccine effectiveness against carriage; VEIPD = vaccine effectiveness against IPD.

Vaccine effectiveness against IPD is based on Andrews et al. [38] and 2014 for all PCV13 serotypes, excluding serotype 3. For serotype 3, this value is scenario specific: in Scenario 1, serotype 3 is assumed to be an NVT; in Scenario 2, this value is derived from Sings et al. [10]; and in Scenario 3, this value is calibrated.

\subsection{Approach to Modeling PCV13 VE Against Serotype 3}

We conducted a series of scenario analyses to assess PCV13's VE against serotype 3. These scenarios included the following:

a. Scenario 1: $V E_{I P D}$ and $V E_{C}$ against serotype 3 are both assumed to be 0 , mimicking serotype 3 as an NVT.

b. Scenario 2: $V E_{I P D}$ against serotype 3 is assumed to be $63.5 \%$ following the booster dose [10]. $V E_{I P D}$ against serotype 3 for the first and second priming doses followed the same procedure as described in Wasserman et al. [29]. $V E_{C}$ against serotype 3 was a calibrated parameter.

c. Scenario 3: $V E_{I P D}$ and $V E_{C}$ against serotype 3 were calibrated parameters.

The combination of these three scenarios provide for a broad perspective on evaluating PCV13 VE against serotype 3.

The model was first calibrated using the assumptions in Scenario 1. Then, to allow for comparison across scenarios, the pre-PCV13-calibrated inputs and initial conditions in Scenario 1 were also used for Scenarios 2 and 3. The post-PCV13calibrated inputs were then reestimated for Scenarios 2 and 3 by initializing the calibration procedure at the start of PCV13's introduction. For each scenario, the model was calibrated using the same observed data as in Wasserman et al. [29]. All other fixed inputs remained the same across scenarios. To assess the goodness of fit for each calibration, the sum of squared deviations between modeled and observed IPD incidence was compared between scenarios. Fit was assessed for all serotypes and serotype 3 IPD incidence separately.

\subsection{Outcomes}

The primary outcomes of the model were the trends in serotype 3 IPD incidence (from here forward referred to as IPD incidence). The study compared modeled annual IPD incidence rates in 2009 (the year preceding PCV13 introduction) and 2017 (the last year of observed data) with observed data. The study also examined the average annual IPD incidence post PCV13 introduction.

The study compared each scenario's outcome to the observed outcome. While all age groups were included in the model parameterization, results are presented for children aged $0-<2$ years as well as adults aged $\geq 65$ years, because these age groups represent the majority of IPD burden (calibration fit trends are presented in Supplementary 
Material for each age group included in the model). Direct protection against serotype 3 IPD $\left(V E_{I P D}\right)$ was therefore evaluated based on the best-fit scenarios for the vaccinated children aged $0-<2$ years, while change in IPD incidence in the group aged $\geq 65$ years was used to examine the existence of indirect protection $\left(V E_{C}\right)$ against serotype 3 .

\section{Results}

Following the calibration procedure for all scenarios, the model found the best fit for observed data across all age groups in the model (both vaccinated and unvaccinated) with the estimated $V E_{I P D}$ and $V E_{C}$ against serotype 3 and goodness-of-fit estimates as presented in Table 2. In Scenario 2, the model estimated $V E_{C}$ against serotype 3 to be $6 \%$ for the booster dose using a fixed $63.5 \% V E_{I P D}$. In Scenario 3, the model estimated $V E_{I P D}$ and $V E_{C}$ against serotype 3 to be $31 \%$ and $19 \%$, respectively. Scenario 3 had the best fit to observed data for all serotypes in addition to serotype 3 .

Table 2. Vaccine Effectiveness Against Serotype 3 for Each Scenario.

\begin{tabular}{lllllll}
\hline \multirow{2}{*}{ Parameter } & Scenario 1 & & & Scenario 2 & \\
\cline { 2 - 7 } & $\begin{array}{l}\text { First Priming } \\
\text { Dose }\end{array}$ & $\begin{array}{l}\text { Second Priming } \\
\text { Dose }\end{array}$ & Booster Dose & $\begin{array}{l}\text { First Priming } \\
\text { Dose }\end{array}$ & $\begin{array}{l}\text { Second Priming } \\
\text { Dose }\end{array}$ & Booster Dose \\
\hline VE against IPD & $0 \%$ & $0 \%$ & $0 \%$ & $41 \%$ & $65 \%$ & $64 \%$ \\
VE against carriage & $0 \%$ & $0 \%$ & $0 \%$ & $4 \%$ & $6 \%$ & $6 \%$ \\
Goodness of fit ${ }^{2}$, all serotypes & & 795.2 & & & 859.9 & 19.1 \\
Goodness of fit ${ }^{2}$, serotype 3 & 31.2 & & & \\
\hline
\end{tabular}

Table 2. Continued.

\begin{tabular}{llll}
\hline \multirow{2}{*}{ Parameter } & Scenario 3 & & \\
\cline { 2 - 4 } & First Priming Dose & Second Priming Dose & Booster Dose \\
\hline VE against IPD & $15 \%$ & $32 \%$ & $31 \%$ \\
VE against carriage & $10 \%$ & $14 \%$ & $19 \%$ \\
Goodness of fit ${ }^{a}$, all serotypes & & 793.0 & \\
Goodness of fit ${ }^{2}$, serotype 3 & & 16.3 & \\
\hline
\end{tabular}

$\mathrm{IPD}=$ invasive pneumococcal disease; $\mathrm{PCV}=$ pneumococcal conjugate vaccine; $\mathrm{VE}=$ vaccine effectiveness.

${ }^{\text {a }}$ Sum of squared deviations between modeled and observed IPD incidence for all serotype groups, age groups, and years.

${ }^{\mathrm{b}}$ Sum of squared deviations between modeled and observed serotype 3 IPD incidence, for all age groups and years.

Figures 1 and 2 illustrate the trends in IPD incidence for each scenario and for the observed data for children aged $0-<2$ years and adults aged $\geq 65$ years, respectively (results of the calibrated parameters and trends in IPD incidence for each age group included in the model are listed in Supplementary Table 1 and Figures 1 to 7). Pre-PCV13 (2001-2009) modeled IPD incidence aligned well with the observed data. From 2010 on, the fit (compared to observed data) and trend lines differed across scenarios due to the calibration. In observed data, IPD incidence decreased after 2009 and then immediately increased
(Figures 1 and 2). After the introduction of PCV13, modeled IPD incidence in Scenario 1 (assuming serotype 3 is an NVT) increased annually for both age groups. Conversely, for children aged $0-<2$ years, scenarios allowing for direct and indirect protection against serotype 3 led to incidence decreasing before leveling off (Scenarios 2 and 3). For adults aged $\geq 65$ years, Scenario 2 showed a lower increase in IPD incidence than Scenario 1 after 2009, and Scenario 3 resulted in IPD incidence roughly plateauing from the point of PCV13 introduction.

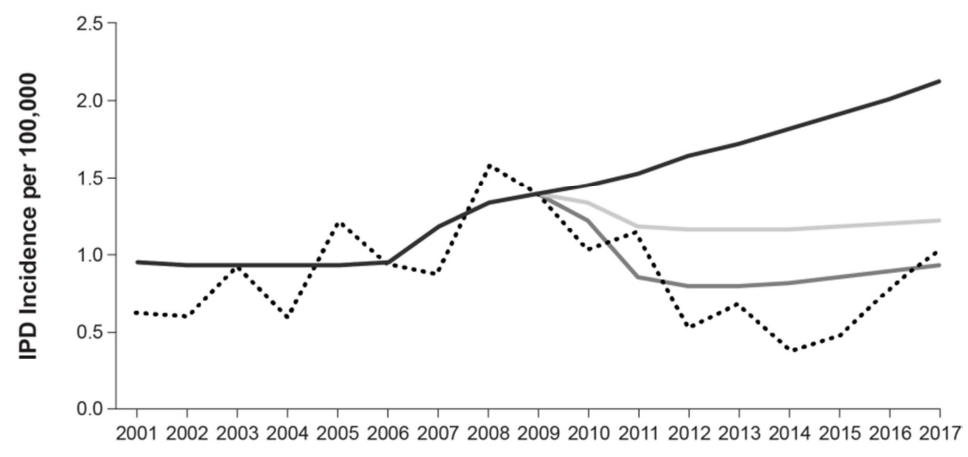

...... Observed surveillance - Scenario $1-$ Scenario $2-$ Scenario 3

Figure 1. Modeled and Observed Serotype 3 IPD Incidence (Cases per 100000) From 2001 to 2017: Children Aged 0-<2 Years.

IPD = invasive pneumococcal disease; PCV13 = 13-valent pneumococcal conjugate vaccine.

Scenario 1 assumes serotype 3 is a nonvaccine serotype. Scenario 2 derives the PCV13 vaccine effectiveness against serotype 3 IPD from Sings et al. [10]. Scenario 3 assumes the PCV13 vaccine effectiveness against both serotype 3 IPD and carriage is unknown, and the model calibrates these values. Year refers to the latter epidemiological year (e.g., epidemiological year 2000/2001 is referred to as 2001). 


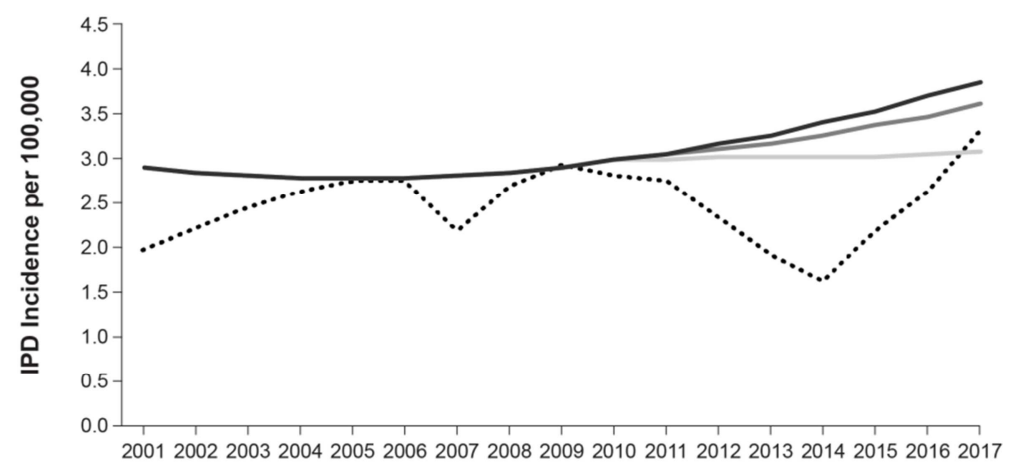

....... Observed surveillance $\longrightarrow$ Scenario $1-$ Scenario $2-$ Scenario 3

Figure 2. Modeled and Observed Serotype 3 IPD Incidence (Cases per 100000) From 2001 to 2017: Adults Aged $\geq 65$ Years.

IPD = invasive pneumococcal disease; PCV13 = 13-valent pneumococcal conjugate vaccine.

Scenario 1 assumes serotype 3 is a nonvaccine serotype. Scenario 2 derives the PCV13 vaccine effectiveness against serotype 3 IPD from Sings et al. [10]. Scenario 3 assumes the PCV13 vaccine effectiveness against both serotype 3 IPD and carriage is unknown, and the model calibrates these values. Year refers to the latter epidemiological year (e.g., epidemiological year 2000/2001 is referred to as 2001).

Table 3 quantifies the results presented in Figures 1 and 2 and presents the modeled IPD incidence in 2009 (pre-PCV13) and 2017 (most recent year of observed data) and the average annual IPD incidence over the entire PCV13 era (2010-2017) for each scenario. In 2009, observed IPD incidence was 1.41 cases per 100,000 among children aged $0-<2$ years (Table 3 ). Modeled IPD incidence in 2009 was within $0.2 \%$ of observed data for the same age group. For children aged $0-<2$ years, following the introduction of PCV13, in Scenario 1, modeled 2017 IPD incidence and the average annual IPD incidence in 2010-2017 deviated from observed IPD incidence by $107.7 \%$ (2.14 vs 1.03 cases per 100000) and $134.2 \%$ (1.79 vs 0.76 cases per 100000 ), respectively. By contrast, in scenarios 2 and 3, for children aged $0-<2$ years, modeled 2017 and average annual IPD incidence remained within roughly $20 \%$ (Scenario 2 ) and $60 \%$ (Scenario 3 ) of observed data. Scenario 2 resulted in the smallest average annual deviation from the observed data in 2017 and in the average annual IPD incidence.

Table 3. Serotype 3 IPD Incidence (Cases per 100000) Over the PCV13 Era.

\begin{tabular}{llll}
\hline \multirow{2}{*}{ Parameter } & \multicolumn{2}{c}{ Serotype 3 IPD Incidence (Cases per 100000) (percentage deviation from observed) } \\
\cline { 2 - 4 } & $\mathbf{2 0 0 9}$ & $\mathbf{2 0 1 7}$ & $\mathbf{2 0 1 0 - 2 0 1 7}($ Average Annual) \\
\hline Children aged 0-<2 years & 1.41 & 1.03 & 0.76 \\
Observed & & $2.14(107.7 \%)$ & $1.79(134.2 \%)$ \\
Scenario 1 & $1.41(-0.2 \%)$ & $0.94(-8.7 \%)$ & $0.90(18.4 \%)$ \\
Scenario 2 & & $1.23(19.5 \%)$ & $1.21(58.7 \%)$ \\
Scenario 3 & 2.93 & 3.34 & 2.45 \\
Adults aged $\geq 65$ years & & $3.88(16.0 \%)$ & $3.38(37.7 \%)$ \\
Observed & $2.92(-0.3 \%)$ & $3.62(8.3 \%)$ & $3.26(32.9 \%)$ \\
Scenario 1 & & $3.09(-7.6 \%)$ & $3.03(23.5 \%)$ \\
Scenario 2 & & \\
Scenario 3 & &
\end{tabular}

IPD = invasive pneumococcal disease; NVT = nonvaccine serotype; PCV = pneumococcal conjugate vaccine; PCV13 = 13 valent PCV.

Relative difference from the observed data (presented in parentheses) is estimated as the relative difference from the observed estimate. For example, a relative difference of $-10 \%$ would suggest that the calibrated estimate is $90 \%$ of that of the observed data, while a relative difference of $100 \%$ would suggest that the calibrated estimate is twice that of the observed data.

For adults aged $\geq 65$ years, observed IPD incidence was 2.93 cases per 100,000 in $2009,3.34$ per 100,000 in 2017 , and an average of 2.45 cases per 100,000 in 2010-2017 (Table 3). Modeled 2017 IPD incidence in Scenario 1 differed from observed data by $16 \%$ in Scenario 1 versus roughly $8 \%$ in Scenarios 2 and 3. When considering the average annual IPD incidence in 2010-2017, the model differed from observed data by roughly $38 \%$ (Scenario 1), $33 \%$ (Scenario 2), and 24\% (Scenario 3).

Observed IPD incidence rate ratios for adults aged $\geq 65$ years from the ECDC $[17,18]$ were also compared with modeled IPD incidence rate ratios from Scenario 1 for the same age group
(Figure 3). Observed data from six sites with universal childhood PCV13 programmes showed a general decrease in serotype 3 IPD incidence rate ratios in 2011-2014, followed by an increase, whereas data from four sites with universal PCV10 programmes showed a general increase in serotype 3 IPD incidence rate ratios starting at the time of PCV10 introduction. When using the modeled IPD incidence rate ratios in Scenario 1 that assume serotype 3 is an NVT, the data were more closely aligned with the observed data from PCV10 sites but underestimated the observed increases. In addition, two PCV10 countries included regions that used PCV13, thus the ECDC data also may have underestimated IPD incidence in PCV10 countries. 


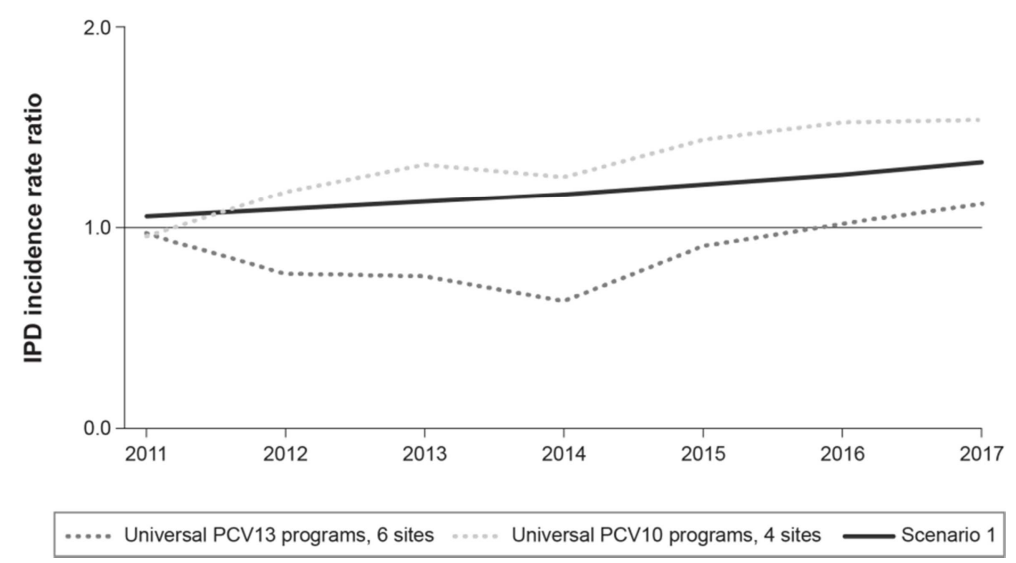

Figure 3. Serotype 3 IPD Incidence Rate Ratios from 2011 to 2017 for Adults Aged $\geq 65$ s: Comparing Serotype 3 as an NVT to ECDC Data.

$\mathrm{ECDC}=$ European Centre for Disease Prevention and Control; IPD = invasive pneumococcal disease; NVT = nonvaccine serotype; PCV10 = 10-valent pneumococcal conjugate vaccine; PCV13 = 13-valent pneumococcal conjugate vaccine; $\mathrm{VE}=$ vaccine effectiveness.

IPD incidence rate ratios compare annual serotype 3 IPD incidence with that of 2009. Scenario 1 assumes serotype 3 is an NVT. Data for universal PCV13 and PCV10 programs taken from Figures $4 \mathrm{a}$ and $4 \mathrm{~b}$ in Hanquet et al. [17], along with data from the February 2019 meeting of the Advisory Committee on Immunization Practices [18]. Year refers to the latter epidemiological year (e.g., epidemiological year 2000/2001 is referred to as 2001).

\section{Discussion}

Using a previously developed dynamic transmission model, we tested several scenario analyses to estimate PCV13 VE against serotype 3 assuming a $2+1$ schedule in the UK. Of the three scenarios tested, modeled results produced the closest approximation to observed IPD incidence among children aged $0-<2$ years when assuming $V E_{I P D}$ against serotype 3 was equal to $63.5 \%$, a value taken from a previous meta-analysis of PCV13 VE against serotype 3 IPD in children [10]. This evidence further suggests that PCV13 provides direct protection against serotype 3 among vaccinated persons.

The evidence for PCV13 indirect protection against serotype 3 was mixed in our findings. Scenarios assuming non-zero $V E_{I P D}$ and $V E_{C}$ (Scenarios 2 and 3) exhibited better alignment with the average observed annual IPD incidence among adults aged $\geq 65$ years post PCV13 introduction. However, these scenarios were not able to fully capture the immediate decrease in IPD incidence in the $\geq 65$-year age group that was observed in the UK. In contrast, assuming serotype 3 is an NVT resulted in markedly higher IPD incidence than was observed during this time period. However, the fits were generally better for the 0 - to $<2$-year population (direct protection) than for the $\geq 65$-year population (indirect protection).

Modeled results for adults aged $\geq 65$ years in Scenarios 2 and 3 were consistent with observed serotype 3 IPD incidence trends in countries with PCV13. Many countries with pediatric PCV13 immunization programmes have experienced a decrease and subsequent increase in serotype 3 IPD incidence among unvaccinated persons aged $\geq 65$ years. If PCV13 does not provide sufficient protection against carriage of serotype 3 , then, over time, cases of serotype 3 could increase if serotype 3 carriage and transmission increase. Conversely, countries that have implemented pediatric PCV10 programmes have experienced an immediate and consistent upward trend in pediatric serotype 3 IPD incidence. As presented in Figure 3, compared to PCV10 countries, PCV13 countries also have experienced a markedly different serotype 3 evolution among unvaccinated older persons. Similarly, comparing the results of the model in Scenario 1 to the ECDC data from PCV10 countries demonstrated that if PCV13VE $V E_{C}$ against serotype 3 were $0 \%$, then IPD incidence rates from PCV13 settings would be much higher and more closely aligned to PCV10 settings. In summary, these results highlight that a nontrivial, possibly low level of indirect protection against serotype 3 is conferred by PCV13 and could reflect impact against carriage acquisition, density, or duration. However, it should also be noted that several serotypes that are known to cause IPD, including serotype 3 , undergo multiyear epidemic patterns that are independent of PCVs [30] and that may be due to a variety of factors. The observed differences in the PCV13 and PCV10 countries could also have been due to factors other than vaccine, including differences in the serotype distribution of nasopharyngeal carriage among children in the pre-PCV10 or PCV13 vaccine periods [31].

Given our model's difficulty in fitting observed incidence in adults aged $\geq 65$ years, it is likely that other nonvaccine exogenous factors play a role in serotype 3 dynamics and NVT replacement in the UK, and several hypotheses exist. First, as with influenza infection, pneumococcal carriage density may increase logarithmically with use of live attenuated influenza vaccine (LAIV), which, in the UK, is the primary influenza vaccine used among young children, the age group most likely to transmit infection [32]. Several lines of evidence are consistent with but do not confirm this hypothesis. A randomized clinical trial of 151 children found that pneumococcal carriage density was 2.68 times higher in children aged 2-4 years who received LAIV compared with those who 
did not [33]. A second study in mice found that LAIV increased bacterial transmigration and could increase the risk of otitis media [34]. Given that the UK has a pediatric influenza vaccine uptake of $60 \%-80 \%$ [32], a relatively modest protection from PCV13 against serotype 3 could be overwhelmed by stimulation of pneumococcal proliferation among heavy transmitters immediately before the season of greatest pneumococcal disease risk. Second, there may be additional carriage reservoirs outside of children that may lead to increases in disease caused by NVT and serotype 3, as vaccine serotype carriage is reduced at a population level. By using pediatric carriage-testing methodology (e.g., a focus on the nasopharynx versus saliva and oropharynx), recent studies have reported that adult pneumococcal carriage has been underestimated multiple-fold [35]. Third, a recent report on global serotype 3 genotypes and genetic evolution reported that a new antimicrobial-resistant clade of serotype 3 emerged in 2014 [36], and that emergence did not correlate with the use or timing of introduction of PCV13 at a population level. As with the LAIV hypothesis, this finding raises the possibility that PCV13 impact is being overwhelmed by a new clade for which community antibiotic pressure is no longer providing sufficient synergy. Fourth, serotype 3 may actually represent a serogroup with multiple related serotypes, similar to those identified in the recent separation of serotypes 6A and 6C [37]. In this scenario, PCV13 may have greater impact against one form of serotype 3 , which then is replaced by a second form less susceptible to vaccineinduced immunity. However, further effectiveness or immunogenicity studies are required to support this theory. Fifth, there may be changes in antibiotic pressure with rational antibiotic use policies, or temporal changes may have occurred in risk factors for pneumococcal disease, including those that favor serotype 3, such as aging of the population, increased prevalence of chronic diseases, changes in breastfeeding or child group care practices, or changes use of extended care facilities for older adults. Finally, there are aspects of pneumococcal disease that are still not fully understood. For example, NVT IPD incidence in the UK increased approximately linearly following the introduction of PCV13 [5]. Starting in 2014, however, NVT incidence substantially increased [5]. Further research is recommended to better understand these phenomena and to better capture the dynamics of a very complex disease.

As with any modeling study, this analysis is subject to several limitations. The most notable limitation is the limited availability of data on VE and duration of protection for each dose. Similarly, real-world data on carriage of each serotype over time is extremely limited. The results are thus subject to uncertainty. A further limitation is that the results are based on only one country, and as such our findings may or may not be generalizable to other settings. This question could be explored in other countries for which PCV13 was introduced to better assess the consistency of evidence of PCV13 against serotype 3 . However, few countries have sufficiently robust data available to model complex serotype dynamics before and after vaccine introduction. Additionally, for computational reasons and lack of data, the model does not consider the impact of immigration/emigration or transient travel on carriage and IPD incidence. These factors could influence the transmission of disease carriage, as individuals traveling from countries without PCV13 may affect the carriage rate within the UK population. Finally, implications of LAIV or other population-level impacts that could be driving increases in serotype 3 IPD incidence in more recent years, as discussed above, were not addressed in the model. These structural limitations would require a substantially more computationally intensive model, and that combined with the absence of data to estimate key parameters may render accounting for them infeasible.

\section{Conclusions}

Using a dynamic transmission model parameterized with the best available evidence and calibrated to the UK surveillance system, our results support the hypothesis that PCV13 provides direct protection against serotype 3 for vaccinated persons and may provide additional protection against some aspect of serotype 3 carriage, whether acquisition, density, or duration. Policymakers should consider direct and indirect effects of conjugate pneumococcal vaccines when interpreting changes in disease incidence rates, including those for specific serotypes. Additionally, policymakers should recognize that PCVs represent only one of many potentially competing factors that can influence pneumococcal disease epidemiology. Further research is necessary to better understand the complexity of disease transmission dynamics and the evolution of serotype epidemiology.

\section{Supplementary Material}

\subsection{Calibrated Parameter Estimates}

All non-calibrated parameters are taken from Wasserman et al., 2018. All parameters that varied across scenarios are listed in Table 4.

Table 4. Calibrated Parameters for Each Scenario.

\begin{tabular}{|c|c|c|c|c|c|c|}
\hline \multirow[b]{2}{*}{ Parameter } & \multicolumn{3}{|l|}{ Scenario 1} & \multicolumn{3}{|l|}{ Scenario 2} \\
\hline & $\begin{array}{l}\text { First Priming } \\
\text { Dose }\end{array}$ & $\begin{array}{l}\text { Second Priming } \\
\text { Dose }\end{array}$ & $\begin{array}{l}\text { Booster } \\
\text { Dose }\end{array}$ & $\begin{array}{l}\text { First Priming } \\
\text { Dose }\end{array}$ & $\begin{array}{l}\text { Second Priming } \\
\text { Dose }\end{array}$ & $\begin{array}{l}\text { Booster } \\
\text { Dose }\end{array}$ \\
\hline \multicolumn{7}{|c|}{ Indirect protection (VE against carriage) } \\
\hline Serotype 19A & $15 \%$ & $22 \%$ & $73 \%$ & $14 \%$ & $34 \%$ & $74 \%$ \\
\hline Serotype 3 & $0 \%$ & $0 \%$ & $0 \%$ & $4 \%$ & $6 \%$ & $6 \%$ \\
\hline Serotypes $1,5,7 \mathrm{~F}$, and $6 \mathrm{~A}$ & $17 \%$ & $47 \%$ & $71 \%$ & $7 \%$ & $21 \%$ & $21 \%$ \\
\hline PCV7-covered serotypes ${ }^{\mathrm{a}}$ & $29 \%$ & $54 \%$ & $79 \%$ & $29 \%$ & $54 \%$ & $79 \%$ \\
\hline
\end{tabular}




\begin{tabular}{|c|c|c|c|c|c|c|}
\hline \multirow[b]{2}{*}{ Parameter } & \multicolumn{3}{|l|}{ Scenario 1} & \multicolumn{3}{|l|}{ Scenario 2} \\
\hline & $\begin{array}{l}\text { First Priming } \\
\text { Dose }\end{array}$ & $\begin{array}{l}\text { Second Priming } \\
\text { Dose }\end{array}$ & $\begin{array}{l}\text { Booster } \\
\text { Dose }\end{array}$ & $\begin{array}{l}\text { First Priming } \\
\text { Dose }\end{array}$ & $\begin{array}{l}\text { Second Priming } \\
\text { Dose }\end{array}$ & $\begin{array}{l}\text { Booster } \\
\text { Dose }\end{array}$ \\
\hline \multicolumn{7}{|c|}{ Indirect protection (VE against carriage) } \\
\hline $\begin{array}{l}\text { Duration of carriage among carriers } \\
(\mathrm{NVT})^{\mathrm{a}}\end{array}$ & \multicolumn{3}{|l|}{2 weeks } & \multicolumn{3}{|l|}{2 weeks } \\
\hline $\begin{array}{l}\text { Probability of IPD given carriage } \\
\text { acquisition }(\mathrm{NVT})^{\mathrm{a}}\end{array}$ & \multicolumn{3}{|c|}{2 per 100000 acquisitions } & \multicolumn{3}{|c|}{2 per 100000 acquisitions } \\
\hline
\end{tabular}

$\mathrm{IPD}=$ invasive pneumococcal disease $\mathrm{PCV}=$ pneumococcal conjugate vaccine; $\mathrm{VE}=$ vaccine effectiveness; $\mathrm{NVT}=$ nonvaccine serotype.

${ }^{\text {a }}$ To allow for comparison across scenarios, the pre-PCV13 calibrated inputs and initial conditions in Scenario 1 were also used for Scenario 2 and 3.

Table 4. Continued.

\begin{tabular}{llll}
\hline \multirow{2}{*}{ Parameter } & Scenario 3 & & \\
\cline { 2 - 4 } & First Priming Dose & Second Priming Dose & Booster Dose \\
\hline Indirect protection (VE against carriage) & & & $47 \%$ \\
\hline Serotype 19A & $28 \%$ & $40 \%$ & $19 \%$ \\
Serotype 3 & $10 \%$ & $14 \%$ & $65 \%$ \\
Serotypes 1, 5, 7F, and 6A & $21 \%$ & $41 \%$ & $79 \%$ \\
PCV7-covered serotypes & $29 \%$ & $54 \%$ & 17 years \\
Duration of immunity (PCV7 and PCV13) & 14 years & 4 years & \\
Duration of carriage among carriers (NVT) $^{\mathrm{a}}$ & 2 weeks & & \\
Probability of IPD given carriage acquisition (NVT) & & \\
\hline
\end{tabular}

$\mathrm{IPD}=$ invasive pneumococcal disease $\mathrm{PCV}=$ pneumococcal conjugate vaccine; $\mathrm{VE}=$ vaccine effectiveness; $\mathrm{NVT}=$ nonvaccine serotype .

${ }^{\text {a }}$ To allow for comparison across scenarios, the pre-PCV13 calibrated inputs and initial conditions in Scenario 1 were also used for Scenario 2 and 3.

\subsection{Modeled and Observed Serotype 3 IPD Incidence for All Age Groups}

Figures 1 to 7 illustrate the trends in IPD incidence for each scenario and for the observed data for each age group included in the model. Results were inconclusive in analyzing the fit and primary outcomes of interest for people aged $2-64$ years, as IPD incidence was very close to zero for this population.

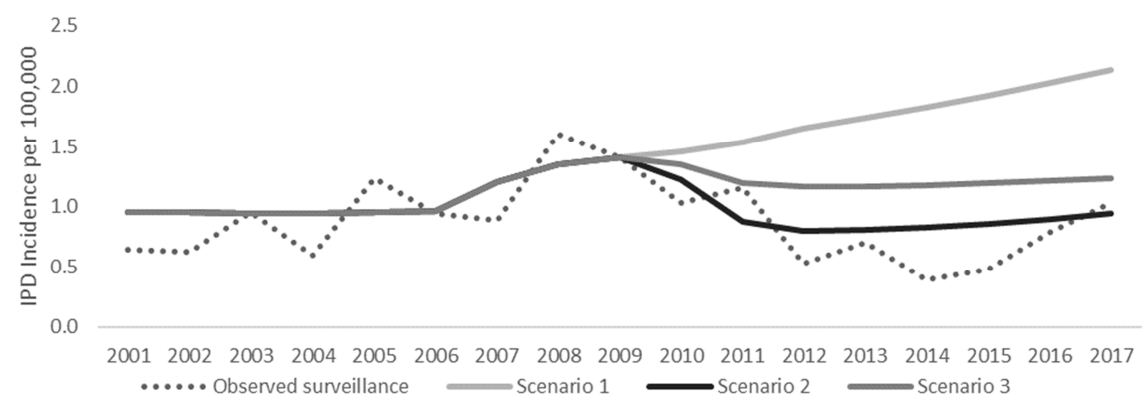

Figure 1. Modeled and Observed Serotype 3 IPD Incidence (Cases per 100000) From 2001 to 2017: 0-<2 Years.

IPD = invasive pneumococcal disease; PCV13 = 13-valent pneumococcal conjugate vaccine.

Scenario 1 assumes serotype 3 is a nonvaccine serotype. Scenario 2 derives the PCV13 vaccine effectiveness against serotype 3 IPD from Sings et al., 2018

[10]. Scenario 3 assumes the PCV13 vaccine effectiveness against both serotype 3 IPD and carriage is unknown, and the model calibrates these values.

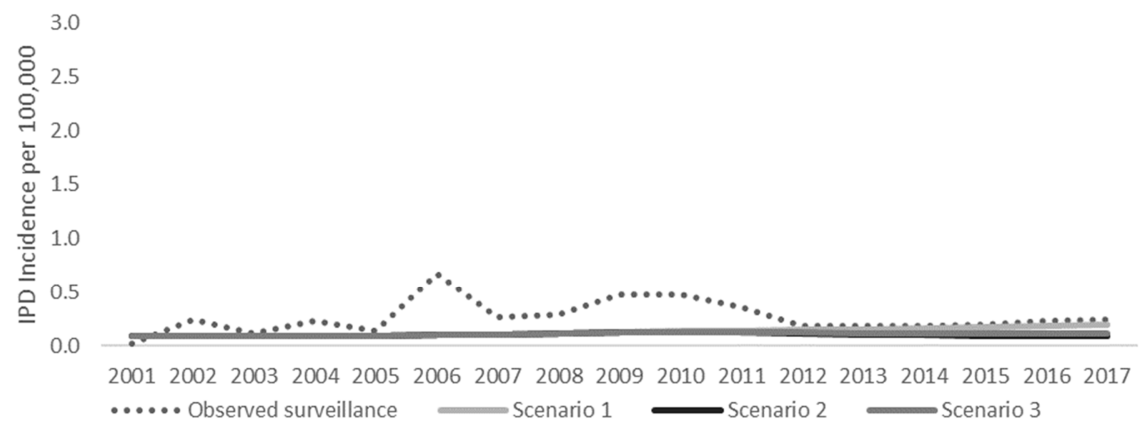

Figure 2. Modeled and Observed Serotype 3 IPD Incidence (Cases per 100000) From 2001 to 2017: 2-<5 Years.

IPD = invasive pneumococcal disease; PCV13 = 13-valent pneumococcal conjugate vaccine.

Scenario 1 assumes serotype 3 is a nonvaccine serotype. Scenario 2 derives the PCV13 vaccine effectiveness against serotype 3 IPD from Sings et al., 2018

[10]. Scenario 3 assumes the PCV13 vaccine effectiveness against both serotype 3 IPD and carriage is unknown, and the model calibrates these values. 


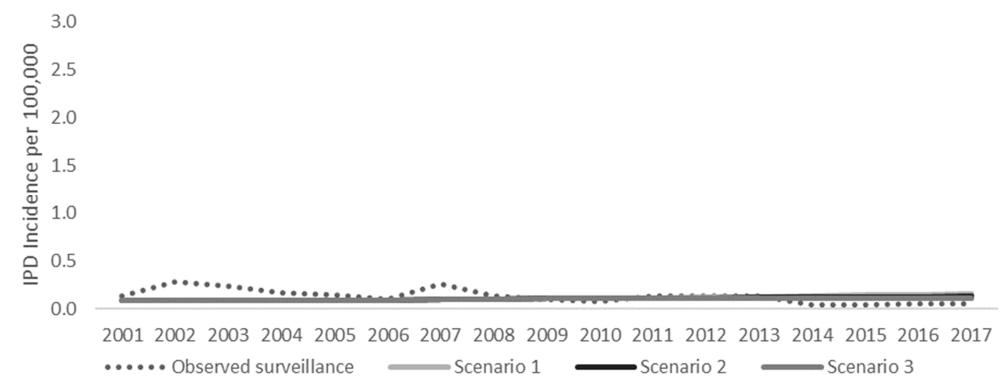

Figure 3. Modeled and Observed Serotype 3 IPD Incidence (Cases per 100000) From 2001 to 2017: 5-<18 Years.

IPD = invasive pneumococcal disease; PCV13 = 13-valent pneumococcal conjugate vaccine

Scenario 1 assumes serotype 3 is a nonvaccine serotype. Scenario 2 derives the PCV13 vaccine effectiveness against serotype 3 IPD from Sings et al., 2018 [10]. Scenario 3 assumes the PCV13 vaccine effectiveness against both serotype 3 IPD and carriage is unknown, and the model calibrates these values.

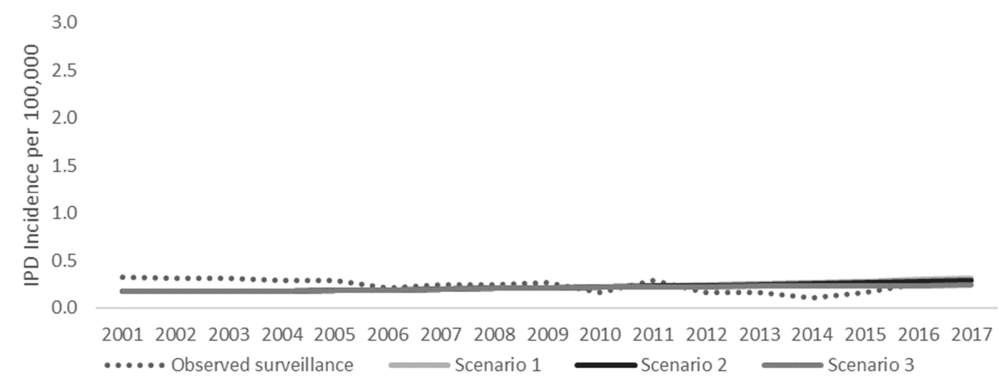

Figure 4. Modeled and Observed Serotype 3 IPD Incidence (Cases per 100000) From 2001 to 2017: 18-<35 Years.

IPD = invasive pneumococcal disease; PCV13 = 13-valent pneumococcal conjugate vaccine.

Scenario 1 assumes serotype 3 is a nonvaccine serotype. Scenario 2 derives the PCV13 vaccine effectiveness against serotype 3 IPD from Sings et al., 2018 [10]. Scenario 3 assumes the PCV13 vaccine effectiveness against both serotype 3 IPD and carriage is unknown, and the model calibrates these values.

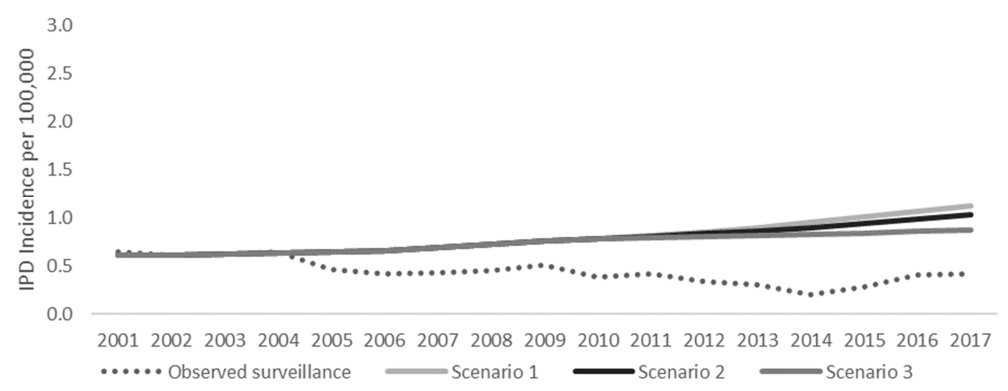

Figure 5. Modeled and Observed Serotype 3 IPD Incidence (Cases per 100000) From 2001 to 2017: 35-<50 Years.

IPD = invasive pneumococcal disease; PCV13 = 13-valent pneumococcal conjugate vaccine.

Scenario 1 assumes serotype 3 is a nonvaccine serotype. Scenario 2 derives the PCV13 vaccine effectiveness against serotype 3 IPD from Sings et al., 2018 [10]. Scenario 3 assumes the PCV13 vaccine effectiveness against both serotype 3 IPD and carriage is unknown, and the model calibrates these values.

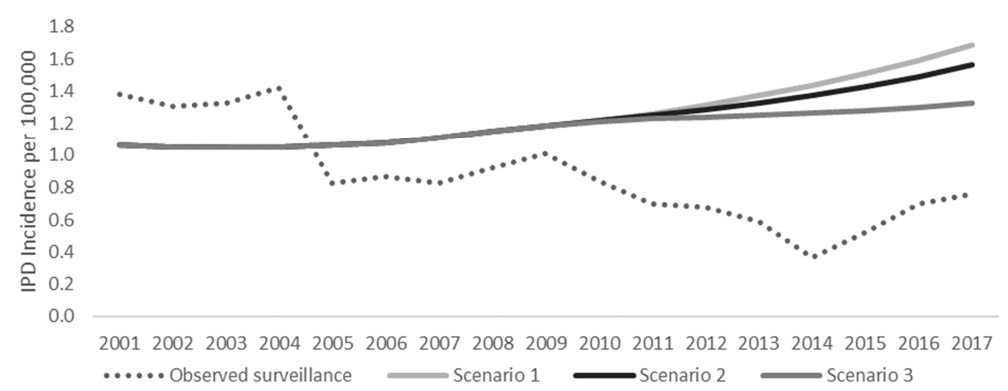

Figure 6. Modeled and Observed Serotype 3 IPD Incidence (Cases per 100000) From 2001 to 2017: 50-<65 Years.

IPD = invasive pneumococcal disease; PCV13 = 13-valent pneumococcal conjugate vaccine.

Scenario 1 assumes serotype 3 is a nonvaccine serotype. Scenario 2 derives the PCV13 vaccine effectiveness against serotype 3 IPD from Sings et al., 2018 [10]. Scenario 3 assumes the PCV13 vaccine effectiveness against both serotype 3 IPD and carriage is unknown, and the model calibrates these values. 


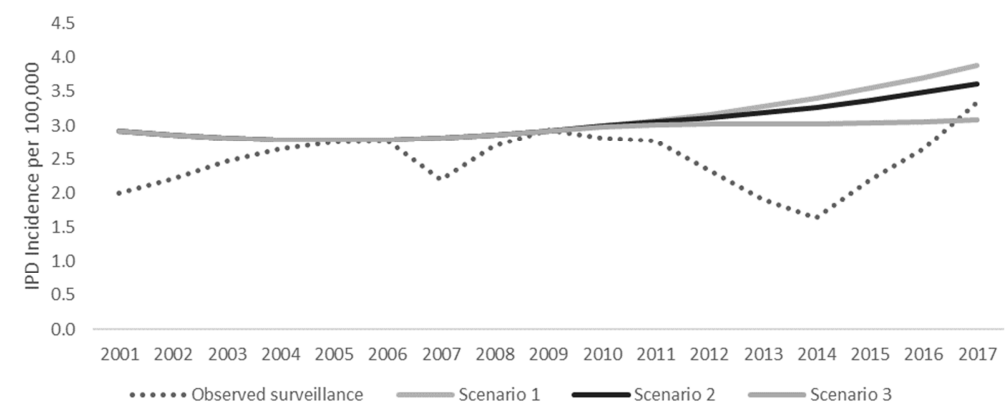

Figure 7. Modeled and Observed Serotype 3 IPD Incidence (Cases per 100000) From 2001 to 2017: $\geq 65$ Years.

IPD = invasive pneumococcal disease; PCV13 = 13-valent pneumococcal conjugate vaccine.

Scenario 1 assumes serotype 3 is a nonvaccine serotype. Scenario 2 derives the PCV13 vaccine effectiveness against serotype 3 IPD from Sings et al., 2018 [[10]]. Scenario 3 assumes the PCV13 vaccine effectiveness against both serotype 3 IPD and carriage is unknown, and the model calibrates these values.

\section{Acknowledgements}

Financial support: This work was funded by Pfizer Inc., Collegeville, United States.

\section{Conflict of Interest}

Dr. Lucas and Mr. Wilson are employees of RTI Health Solutions. Mr. Wasserman, Dr. Sings, Dr. Pugh, Dr. Gessner, and Dr. Farkouh are employees of Pfizer Inc. Dr. Jones is an employee of Pfizer Ltd.

\section{References}

[1] World Health Organization. Expert Committee on Biological Standardization, Geneva, 19 to 23 October 2009. Recommendations to assure the quality, safety and efficacy of pneumococcal conjugate vaccines. Replacement of: TRS 927, Annex 2 [Internet] Geneva: World Health Organization; c2009. [cited 2013 Aug 5]. Available from: http://www.who.int/biologicals/areas/vaccines/pneumo/Pneum o_final_23APRIL_2010.pdf?ua $=1$.

[2] Shiri T, Datta S, Madan J, Tsertsvadze A, Royle P, Keeling $\mathrm{MJ}$, et al. Indirect effects of childhood pneumococcal conjugate vaccination on invasive pneumococcal disease: a systematic review and meta-analysis. Lancet Glob Health. 2017; 5: e51-e59.

[3] Andrews NJ, Waight PA, Burbidge P, Pearce E, Roalfe L, Zancolli $M$, et al. Serotype-specific effectiveness and correlates of protection for the 13-valent pneumococcal conjugate vaccine: a postlicensure indirect cohort study. Lancet Infect Dis. 2014; 14: 839-846.

[4] Wahl B, O'Brien KL, Greenbaum A, Majumder A, Liu L, Chu $\mathrm{Y}$, et al. Burden of Streptococcus pneumoniae and Haemophilus influenzae type b disease in children in the era of conjugate vaccines: global, regional, and national estimates for 2000-15. Lancet Glob Health. 2018; 6: e744-e757.

[5] Ladhani SN, Collins S, Djennad A, Sheppard CL, Borrow R, Fry NK, et al. Rapid increase in non-vaccine serotypes causing invasive pneumococcal disease in England and Wales, 2000-17: a prospective national observational cohort study. Lancet Infect Dis. 2018; 18: 441-451.
[6] Weinberger $R$, van der Linden $M$, Imöhl $M$, von Kries $R$. Vaccine effectiveness of PCV13 in a $3+1$ vaccination schedule. Vaccine. 2016; 34 (18): 2062-2065.

[7] Moore MR, Moore MR, Link-Gelles R, Schaffner W, Lynfield R, Holtzman C, Harrison LH, et al. Effectiveness of 13-valent pneumococcal conjugate vaccine for prevention of invasive pneumococcal disease in children in the USA: a matched case-control study. Lancet Respir Med. 2016; 4: 399-406.

[8] Slotved HC, Dalby T, Harboe ZB, Valentiner-Branth P, Casadevante VF, Espenhain L, et al. The incidence of invasive pneumococcal serotype 3 disease in the Danish population is not reduced by PCV-13 vaccination. Heliyon. 2016; 2: e00198.

[9] Waight PA, Andrews NJ, Ladhani SN, Sheppard CL, Slack MP, Miller E. Effect of the 13-valent pneumococcal conjugate vaccine on invasive pneumococcal disease in England and Wales 4 years after its introduction: an observational cohort study. Lancet Infect Dis. 2015; 15: 535-543.

[10] Sings HL, De Wals P, Gessner BD, Isturiz R, Laferriere C, McLaughlin JM, et al. Effectiveness of 13-valent pneumococcal conjugate vaccine against invasive disease caused by serotype 3 in children: a systematic review and meta-analysis of observational studies. Clin Infect Dis. 2019 May 30; 68 (12): 2135-2143.

[11] Savulescu C, Hanquet G; SpIDnet Group. PCV13 effectiveness and overall effect of PCV10/13 vaccination programmes in children under five years of age. SpIDnet multicentre studies. ESCAIDE 2016; Available from: https://www.slideshare.net/ECDC_EU/camelia-savulescupcv13-effectiveness-and-overall-effect-of-pcv1013vaccination-programmes-in-children-under-five-years-of-agespidnet-multicentre-studies?qid $=c 8833740-53 \mathrm{~d} 0-4 \mathrm{c} 03-8163$ $533 \mathrm{f} 54 \mathrm{e} 86 \mathrm{~d} 29 \& \mathrm{v}=\& \mathrm{~b}=\&$ from_search $=2$. Accessed July $22 \mathrm{nd}$, 2018.

[12] Bonten MJ, Huijts SM, Bolkenbaas M, Webber C, Patterson $\mathrm{S}$, Gault S, et al. Polysaccharide conjugate vaccine against pneumococcal pneumonia in adults. N Engl J Med. 2015; 372: 1114-1125.

[13] Gessner BD, Jiang Q, Van Werkhoven CH, Sings HL, Webber C, Scott D, et al. A post-hoc analysis of serotype-specific vaccine efficacy of 13-valent pneumococcal conjugate vaccine against clinical community acquired pneumonia from a randomized clinical trial in the Netherlands. Vaccine. $2019 \mathrm{Jul}$ 9; 37 (30): 4147-4154. 
[14] McLaughlin JM, Jiang Q, Gessner BD, Swerdlow DL, Sings $\mathrm{HL}$, Isturiz RE, et al. Pneumococcal conjugate vaccine against serotype 3 pneumococcal pneumonia in adults: A systematic review and pooled analysis. Vaccine. 2019 Oct 8; 37 (43): 6310-6316.

[15] Andrews N, Kent A, Amin-Chowdhury Z, Sheppard C, Fry N, Ramsay $M$, et al. Effectiveness of the seven-valent and thirteen-valent pneumococcal conjugate vaccines in England: The indirect cohort design, 2006-2018. Vaccine. 2019 Jul 26; 37 (32): 4491-4498.

[16] Prevenar 13. Summary of product characteristics. Available from: http://www.ema.europa.eu/docs/en GB/document library/EPAR _-_Product_Information/human/001104/WC500057247.pdf.

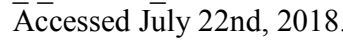

[17] Hanquet G, Krizova P, Valentiner-Branth P, Ladhani SN, Nuorti JP, Lepoutre A, et al. Effect of childhood pneumococcal conjugate vaccination on invasive disease in older adults of 10 European countries: implications for adult vaccination. Thorax. 2019; 74: 473-482.

[18] Pilishvili T. 13-valent pneumococcal conjugate vaccine (PCV13) effects on disease caused by serotype 3. Presented at: Meeting of the Advisory Committee on Immunization Practices; February 28, 2019; Atlanta, Georgia.

[19] Dagan R, Patterson S, Juergens C, Greenberg D, Givon-Lavi $\mathrm{N}$, Porat N, et al. Comparative immunogenicity and efficacy of 13-valent and 7-valent pneumococcal conjugate vaccines in reducing nasopharyngeal colonization: a randomized doubleblind trial. Clin Infect Dis. 2013; 57: 952-962.

[20] Southern J, Andrews N, Sandu P, Sheppard CL, Waight PA, Fry NK, et al. Pneumococcal carriage in children and their household contacts six years after introduction of the 13valent pneumococcal conjugate vaccine in England. PLoS One. 2018; 13: e0195799. doi: 10.1371/journal.pone.0195799.

[21] Izurieta P, Bahety P, Adegbola R, Clarke C, Hoet B. Public health impact of pneumococcal conjugate vaccine infant immunization programs: assessment of invasive pneumococcal disease burden and serotype distribution. Expert Rev Vaccines. 2018; 17: 479-493.

[22] Joint Committee on Vaccination and Immunisation. Minutes of the meeting on 01 October 2017.

[23] Delgleize E, Leeuwenkamp O, Theodorou E, Van de Velde N. Cost-effectiveness analysis of routine pneumococcal vaccination in the UK: a comparison of the $\mathrm{PHiD}-\mathrm{CV}$ vaccine and the PCV-13 vaccine using a Markov model. BMJ Open. 2016; 6: e010776.

[24] Zhang X-H, Leeuwenkamp O, Oh KB, Lee YE, Kim CM. Cost-effectiveness analysis of infant pneumococcal vaccination with PHiD-CV in Korea. Hum Vaccin Immunother. 2018; 14: 85-94.

[25] Shiragami M, Mizukami A, Leeuwenkamp O, Mrkvan T, Delgleize E, Kurono Y, et al. Cost-effectiveness evaluation of the 10-valent pneumococcal non-typeable Haemophilus influenzae protein $\mathrm{D}$ conjugate vaccine and 13-valent pneumococcal vaccine in Japanese children. Infect Dis Ther. 2015; 4: 93-112.
[26] Armbruster B, Lucas AM. Effectiveness of a no-sex or safesex month in reducing HIV transmission. Bull World Health Organ. 2012; 90: 504-512.

[27] Brisson M, Melkonyan G, Drolet M, De Serres G, Thibeault $\mathrm{R}$, De Wals P. Modeling the impact of one-and two-dose varicella vaccination on the epidemiology of varicella and zoster. Vaccine. 2010; 28: 3385-3397.

[28] Granich RM, Gilks CF, Dye C, De Cock KM, Williams BG. Universal voluntary HIV testing with immediate antiretroviral therapy as a strategy for elimination of HIV transmission: a mathematical model. Lancet. 2009; 373: 48-57.

[29] Wasserman M, Lucas A, Jones D, Wilson M, Hilton B, Vyse A, Madhava $\mathrm{H}$, et al. Dynamic transmission modelling to address infant pneumococcal conjugate vaccine schedule modifications in the UK. Epidemiol Infect. 2018; 146: 17971806.

[30] Weinberger DM, Warren JL, Dalby T, Shapiro ED, ValentinerBranth P, Slotved HC, et al. Differences in the impact of pneumococcal serotype replacement in individuals with and without underlying medical conditions. Clin Infect Dis. 2019 Jun 18; 69 (1): 100-106.

[31] Lewnard JA, Hanage WP. Making sense of differences in pneumococcal serotype replacement. Lancet Infect Dis. 2019 Jun; 19 (6): e213-e220.

[32] de Lusignan S, Correa A, Ellis J, Pebody R. Influenza vaccination: in the UK and across Europe. Br J Gen Pract. 2016; 66: 452-453.

[33] Thors V, Christensen H, Morales-Aza B, Vipond I, Muir P, Finn A. The effects of live attenuated influenza vaccine on nasopharyngeal bacteria in healthy 2 to 4 year olds. A randomized controlled trial. Am J Respir Crit Care Med. 2016; 193: 1401-1409.

[34] Mina MJ, Klugman KP, Rosch JW, McCullers JA. Live attenuated influenza virus increases pneumococcal translocation and persistence within the middle ear. J Infect Dis. 2015; 212: 195-201.

[35] Trzciński K, Bogaert D, Wyllie A, Chu ML, van der Ende A, Bruin JP, et al. Superiority of trans-oral over trans-nasal sampling in detecting Streptococcus pneumoniae colonization in adults. PloS One. 2013; 8: e60520.

[36] Azarian T, Mitchell PK, Georgieva M, Thompson CM, Ghouila A, Pollard AJ, et al. Global emergence and population dynamics of divergent serotype 3 CC180 pneumococci. PLoS Pathog. 2018; 14: e1007438.

[37] Park IH, Pritchard DG, Cartee R, Brandao A, Brandileone MC, Nahm MH. Discovery of a new capsular serotype (6C) within serogroup 6 of Streptococcus pneumoniae. J Clin Microbiol. 2007; 45: 1225-1233.

[38] Andrews N, Waight PA, Borrow R, Ladhani S, George RC, Slack MP, et al. Using the indirect cohort design to estimate the effectiveness of the seven valent pneumococcal conjugate vaccine in England and Wales. PloS One. 2011; 6: e28435. 